\title{
Wide/narrow azimuth acquisition footprints and their effects on seismic imaging
}

\author{
Di Bangrang ${ }^{1,2,3} *$, Xu Xiucang ${ }^{1,2,3}$ and Wei Jianxin ${ }^{1,2,3}$ \\ ${ }^{1}$ Key Laboratory of CNPC Geophysical Exploration, China University of Petroleum, Beijing 102249, China \\ ${ }^{2}$ State Key Laboratory of Petroleum Resource and Prospecting, China University of Petroleum, Beijing 102249, China \\ ${ }^{3}$ Key Laboratory of Earth Prospecting and Information Technology, China University of Petroleum, Beijing 102249, China
}

\begin{abstract}
Acquisition footprint is a new concept to describe the seismic noise in three-dimensional seismic exploration and it is closely related to geometry and observation shuttering. At present, the study on acquisition footprints has become a hot spot. In partnership with the Dagang Oilfield, we used the channel sand body seismic physical model to study the characteristics of wide/narrow azimuth acquisition footprints and analyzed and compared the two types of footprints and their effects on target imaging. In addition, the footprints caused by data processing of the normal moveout offset (NMO) stretching aberration were discussed. These footprints are located only in the shallow or middle layer in the time slice, and possibly affect the imaging of shallow target layers, and have no influence on deep target imaging. Seismic physical modeling has its advantages in the study of acquisition footprints.
\end{abstract}

Key words: Wide/narrow azimuth geometry, acquisition footprint, stretching aberration, time slice, seismic imaging

\section{Introduction}

Acquisition footprint is a new concept to describe the seismic noise in three-dimensional (3D) seismic exploration. With the development of high-precision $3 \mathrm{D}$ seismic exploration, the study of acquisition footprint attracts more and more attention both at home and abroad (Marfurt et al, 1998; Canning and Gardner, 1998; Schuster and Liu, 2001; Monk, 1999; Yang, 2003; Feng et al, 2006; Qian et al, 2005; Ma et al, 2008; Tian et al, 2007; Zhang et al, 2007a; $2007 b$ ). In recent years, a number of influential papers have been published. In these papers, acquisition footprint is defined as "any pattern of noise that is highly correlated to the geometric distribution of sources and receivers on the earth's surface" (Marfurt et al, 1998; Dong et al, 2007), or is interpreted as follows: "Incomplete sampling, determined by rolling arrangement as well as line-spacing between source and receiver, will cause periodic illusions of amplitude in the seismic imaging. The illusions of amplitude which usually can be seen in the time and depth sections are acquisition footprints" (Yang, 2003; Hou et al, 2007). Inspired by these, Chinese scientists started to pay attention to acquisition footprints at the beginning of this century and observed the distribution of acquisition footprints in the time slice of actual 3D data. However, since the actual geological structure is unknown, further study is still very difficult but seismic physical simulation technology has great advantages in studying acquisition footprints. In the seismic physical simulation, the geological model is known,

*Corresponding author. email: dibr@cup.edu.cn

Received March 10, 2008 so all relevant information of the geological target can be correctly identified, and the rest is noise. In the residual noise, the acquisition footprint is periodic regular noise, and its regularities are related to the geometry, so it is also known and can be predicted and identified.

In partnership with the Dagang Oilfield Company, PetroChina, our laboratory studied the geological formation mechanism of acquisition footprints and their effects on geological target imaging (Xiong, 2006). Different 3D geometry generates different acquisition footprints. In the past few years, optimization design of wide/narrow azimuth geometry has been a hot issue in the study of high-precision 3D seismic exploration. With the channel sand body seismic physical model, two kinds of geometries (wide/narrow azimuth geometries) were adopted for data acquisition and processing, in order to analyze and compare the impact of these two kinds of footprints on the channel sand imaging.

\section{Seismic physical simulation of channel sand body model}

The channel sand body seismic physical model is composed of a water layer, a matching layer, a sand layer and a basement. Its length, width and height are $730 \mathrm{~mm} \times 504 \mathrm{~mm}$ $\times 254 \mathrm{~mm}$, respectively. Fig. 1 is a vertical view of the model and the vertical slice of west-east centerline. The scale is $1: 10000$, so it is equivalent to the actual geological model of $7,300 \mathrm{~m} \times 5,040 \mathrm{~m} \times 2,540 \mathrm{~m}$. Since the sand layer is solid, while the water layer is liquid, the density difference between them is large. As a result, it is easy to produce a strong reflection at the solid-liquid interface (referred to as water-bottom 

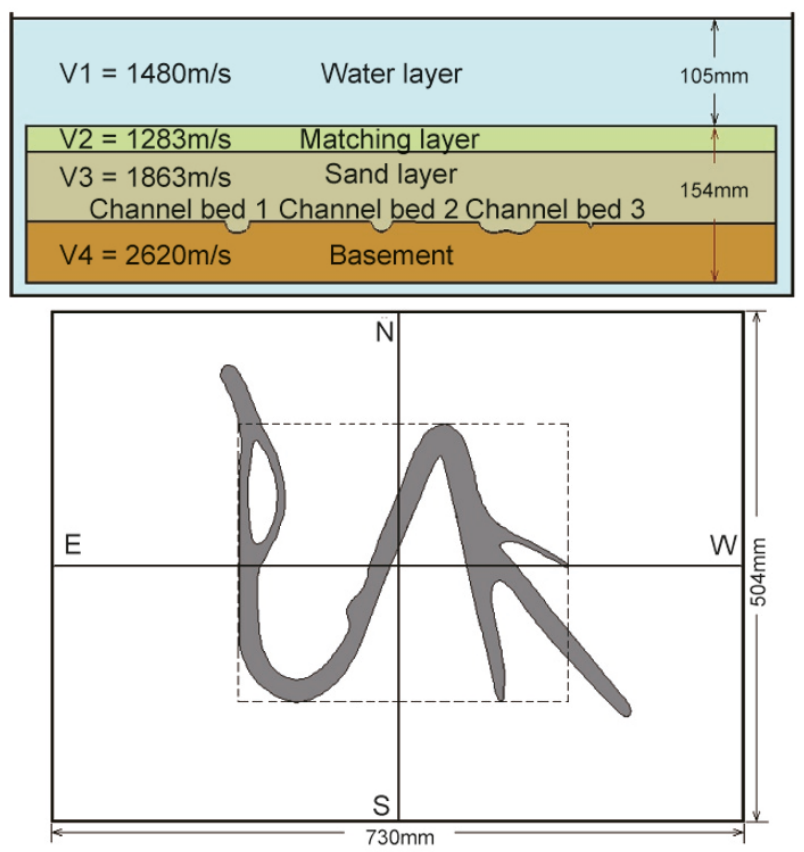

Fig. 1 Vertical slice and vertical view of the channel sand body seismic physical model

reflection). Therefore, we need to design a low-velocity matching layer on the top of the solid layer. The depth of the water layer is $105 \mathrm{~mm}$, and the double-way reflection time is approximately $1,300 \mathrm{~ms}$. The geological target is an ancient stream channel, and its burial depth is $2,000 \mathrm{~m}$. Located at $2,500 \mathrm{~ms}$ in the time slice, the channel width is from $40 \mathrm{~m}$ to $300 \mathrm{~m}$, and the depth of the channel bed is about $40-120 \mathrm{~m}$.

For the same exploration area, different 3D geometries can generate different acquisition footprints. In order to prove this phenomenon, we designed two totally different geometries (wide/narrow azimuth geometries) to make a comparison under the precondition that the sizes of CMP (common middle point) bins are both $20 \mathrm{~m} \times 20 \mathrm{~m}$.

(1) Wide azimuth 16 lines 5 shots $40 \times 2$ traces $8 \times 8$ coverage $3 \mathrm{D}$ geometry

Longitudinal: The inline direction is assumed west-east; alignment interval $200 \mathrm{~m}$; alignment pattern $40-160-1,720 \mathrm{~m}$ ( $40 \mathrm{~m}$ is the group interval, $160 \mathrm{~m}$ is the near sourcereceiver offset, $1,720 \mathrm{~m}$ is the far source-receiver offset); shuttering rolling distance $200 \mathrm{~m}$. From east to west (large source observation shuttering) and from west to east (small source observation shuttering), the shuttering rolls 29 times, respectively with eight coverages in the longitudinal direction each time.

Horizontal: The shot line direction is assumed north-south, shot interval $40 \mathrm{~m}$, shuttering rolling distance $200 \mathrm{~m}$, totally 27 lines and eight coverages in the horizontal direction.

Aspect ratio: The shuttering aspect ratio $\gamma_{\mathrm{t}}$ is 0.904 and coverage aspect ratio $\gamma_{\mathrm{f}}$ is $1, \gamma=\left(\gamma_{\mathrm{t}}+\gamma_{\mathrm{f}}\right) / 2=0.95>0.8$. This is wide azimuth observation (Mou and Pei, 2005).

Fig. 2(a) shows wide azimuth 16 lines 5 shots observation shuttering and $3 \mathrm{D}$ geometry.

(2) Narrow azimuth 8 lines 5 shots $48 \times 2$ traces $4 \times 16$ coverage $3 \mathrm{D}$ geometry

Longitudinal: The inline direction is assumed west-east; alignment interval $200 \mathrm{~m}$; alignment pattern $40-160-2,040 \mathrm{~m}$ ( $40 \mathrm{~m}$ is the group interval, $160 \mathrm{~m}$ is the near sourcereceiver offset, $2,040 \mathrm{~m}$ is the far source-receiver offset); shuttering rolling distance $120 \mathrm{~m}$. From east to west (large source observation shuttering) and from west to east (small source observation shuttering), the shuttering rolls 50 times, respectively with sixteen coverages in the longitudinal direction each time.

Horizontal: The shot line direction is assumed northsouth, shot interval $40 \mathrm{~m}$, shuttering rolling distance $200 \mathrm{~m}$, totally 26 lines and four coverages in the horizontal direction.

Aspect ratio: The shuttering aspect ratio $\gamma_{\mathrm{t}}$ is 0.392 and coverage aspect ratio $\gamma_{\mathrm{f}}$ is $0.25, \gamma=\left(\gamma_{\mathrm{t}}+\gamma_{\mathrm{f}}\right) / 2=0.32<0.5$. This is narrow azimuth observation (Mou and Pei, 2005).

Fig. 2(b) shows narrow azimuth 8 lines 5 shots observation shuttering and 3D geometry.

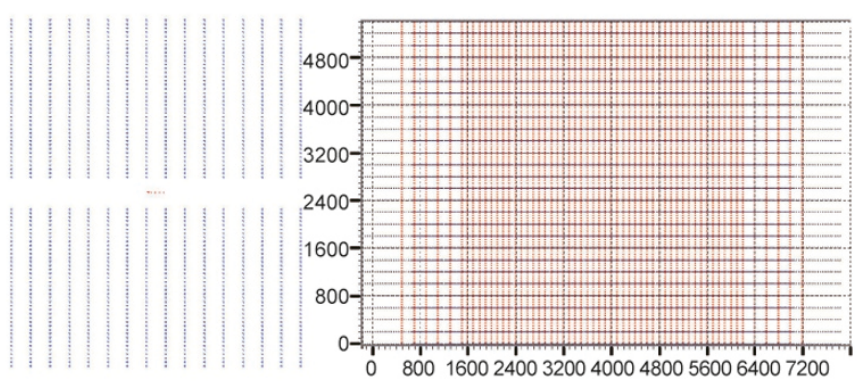

(a) Wide azimuth 16 lines 5 shots

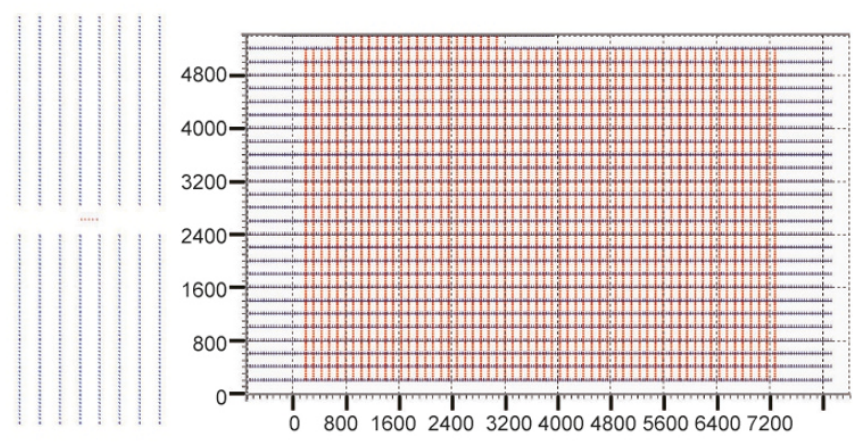

(b) Narrow azimuth 8 lines 5 shots

Fig. 2 Observation shuttering and 3D geometry

The 3D geometry in Fig. 2 is produced by a preprocessing program (the red line in Fig. 2 is the shot line formed by observing shuttering rolling in the longitudinal and horizontal directions, while the blue line is the receiver line also formed by observing shuttering rolling in the longitudinal and horizontal directions). Since the longitudinal rolling distance of the narrow azimuth observation is $120 \mathrm{~m}$, the distribution of shot lines in the geometry figure is concentrated. The longitudinal rolling distance of the wide azimuth observation is $200 \mathrm{~m}$. In principle the distribution of shot lines in the geometry figure should be sparse. Then why is the actual distribution of shot lines concentrated in the figure? To weaken the acquisition footprints, in actual data acquisition, we used the equivalent both ends shooting method instead of the center shooting method. Moreover, the east-to-west shot line and the west-to-east shot line were both moved away 
from each other for $100 \mathrm{~m}$. Then we got the $100 \mathrm{~m}$-interval wide azimuth distribution of shot lines shown in Fig. 2.

We finished the seismic physical simulation data acquisition of wide/narrow azimuth geometries according to the designed project of channel sand body seismic physical model. Wide azimuth acquisition has 4.36 million data traces, while narrow azimuth acquisition has 4.21 million data traces. The workload of both geometries is almost the same, and the full coverages of CMP bins are both 64, which could meet the requirement of comparison of these two kinds of acquisition footprints. After processing, we obtained two sets of 3D migrated data volume. Then we analyzed and compared the footprints by using the horizontal time slice of $3 \mathrm{D}$ migrated data volume.

\section{Analysis of shallow water-bottom acquisition footprints}

The generation mechanism of acquisition footprints is as follows: (1) The discrete distribution of surface shot points and receivers leads to the non-uniform distribution of lighting intensity of the underground horizontal layers, which induces the non-uniform distribution of horizontal stack, migrated amplitude and phase of CMP bins. (2) The regularity of longitudinal and horizontal rolling of 3D observation shuttering creates the periodic variation of the underground CMP bins' attribute character which leads to the periodic variation of the horizontal stack, migrated amplitude and phase of CMP bins. These two mechanisms are universal. Moreover, there is a particular kind of acquisition footprint, which is related to NMO processing in the shallow seismic slice and is analyzed below.

The CMP gather is composed of multi-trace CMP records whose offsets are different. We need to do NMO to each CMP record before stacking in order to achieve co-phasal stacks. A stretching muting factor is designed in the NMO processing program because NMO can create stretching aberration of seismic records and the record whose stretching aberration exceeds the limit will be muted. Generally speaking, the muting does not affect the small offset records and the shallow parts of middle offset records are muted in different degrees. However, the shallow parts of large offset records are totally muted. Apparently, after NMO the stacking fold of CMP bins becomes a function changing with the travel time (depth). Both middle and deep layer stacks are full coverage stacks and both shallow-middle and shallow layer stacks are non-full coverage stacks. Furthermore, with reducing travel time (towards the shallow layer), stacking fold reduces gradually as well. Though the coverage of underground CMP bins is full and uniform, the stacking fold of CMP bins in the shallow layers becomes non-uniform after NMO, which results in the non-uniform distribution of stack amplitude. This phenomenon generates the shallow acquisition footprints related to processing.

Using a 3D geometry designing program, we simulated the acquisition footprints generated by NMO processing. In the given geological model, the depth of water-bottom is $1,050 \mathrm{~m}$, and the reflection travel time is about $1.3 \mathrm{~s}$. We do stretching muting processing for the two geometries. When the largest offset is limited to $1,050 \mathrm{~m}$, the seismic traces whose offset is over $1,050 \mathrm{~m}$ in the CMP gather are removed. The remaining traces are stacked horizontally at the moment of 1.3s. Since the distribution of offset in CMP bins is different, the number of removed traces is also different. Then we obtained the acquisition footprints shown in Fig. 3. The characteristics of acquisition footprint in the wide azimuth geometry is as follows: In narrow band, acquisition footprint is low-amplitude, low coverage (12-16) and its width is $40 \mathrm{~m}$ (two bins). In wide band, the acquisition footprint is high-amplitude, high coverage (17-20) and its width is $80 \mathrm{~m}$ (eight bins). The period of the footprint is $200 \mathrm{~m}$ (ten bins), which is just equal to the longitudinal rolling distance of shuttering. The ratio of high-amplitude to lowamplitude is $18.5: 14.5=1.3$. Apparently, this kind of noise will seriously affect the imaging quality of shallow geological targets. The characteristics of acquisition footprints in the narrow azimuth geometry is as follows: In narrow band, the acquisition footprint is low-amplitude, low coverage (22-24) and its width is $20 \mathrm{~m}$ (one bin). In wide band, the acquisition footprint is high-amplitude, high coverage (27-29) and its width is $100 \mathrm{~m}$ (five bins). The period of the footprint is $120 \mathrm{~m}$ (six bins), which is also just equal to the longitudinal rolling
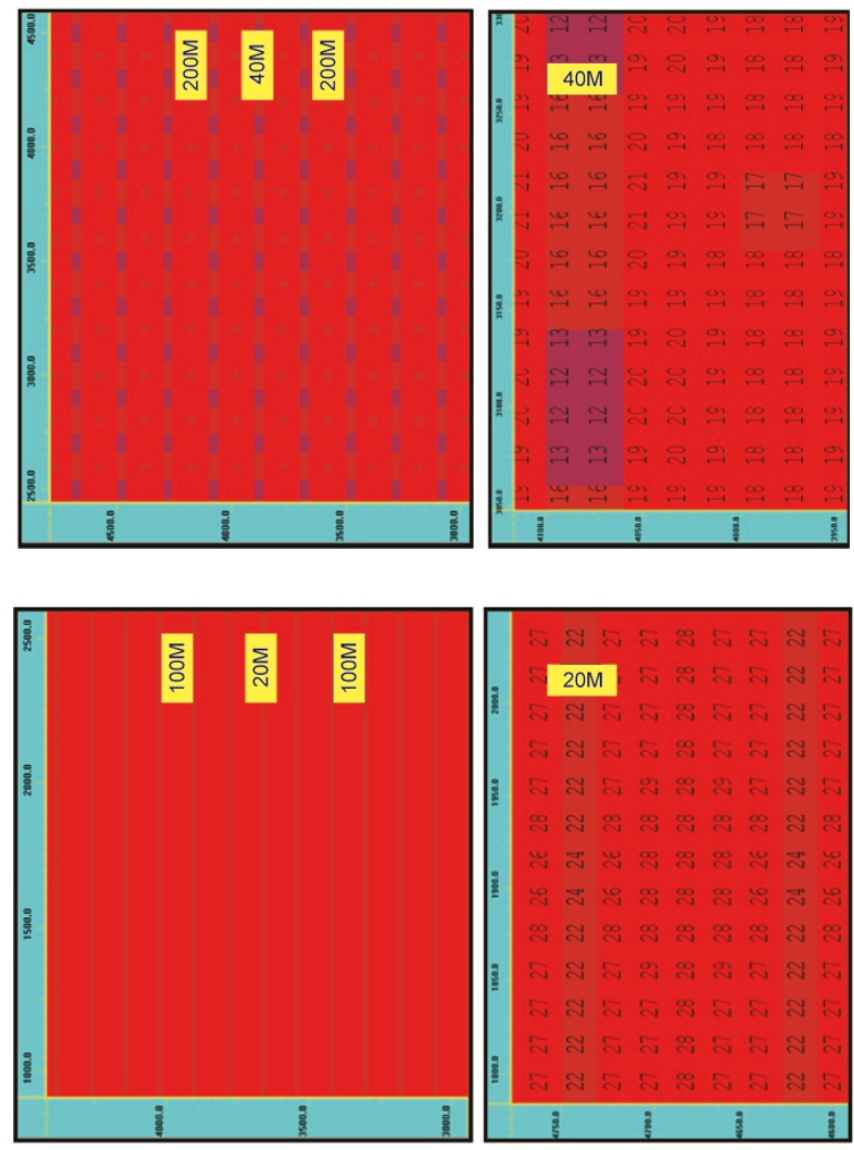

Fig. 3 Acquisition footprints with the largest offset limited to $1050 \mathrm{~m}$ (left figure) and the stacking fold distribution after image magnification (right figure). The figure above is wide azimuth geometry and the figure below is narrow azimuth geometry. 
distance of shuttering. The ratio of high-amplitude to lowamplitude is $28: 22=1.3$. The noise level of narrow azimuth is similar to that of wide azimuth. Therefore, this kind of noise will also seriously affect the imaging quality of shallow geological targets.

Fig. 4 shows the water-bottom reflection time slice acquired from the seismic physical simulation experiment. The figure above shows the acquisition footprints of wide azimuth geometry $(1,352 \mathrm{~ms})$; the figure below shows the acquisition footprints of narrow azimuth geometry $(1,358 \mathrm{~ms})$. The part shown in the red square frame is the acquisition footprints generated by NMO stretching muting. The belt width of the time slice above is $200 \mathrm{~m}$ (the distance between the two white belts), and that in the figure below is $120 \mathrm{~m}$, which are corresponding to the results of software simulation. The part shown in the yellow square frame is the acquisition footprints generated by the stack of east-to-west rolling and

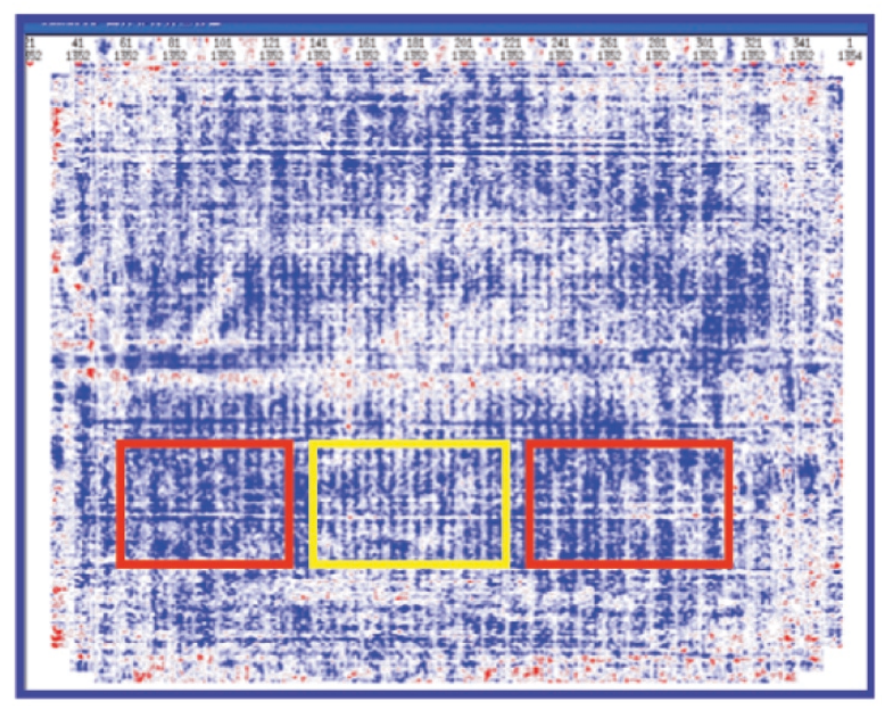

Wide azimuth $1352 \mathrm{~ms}$

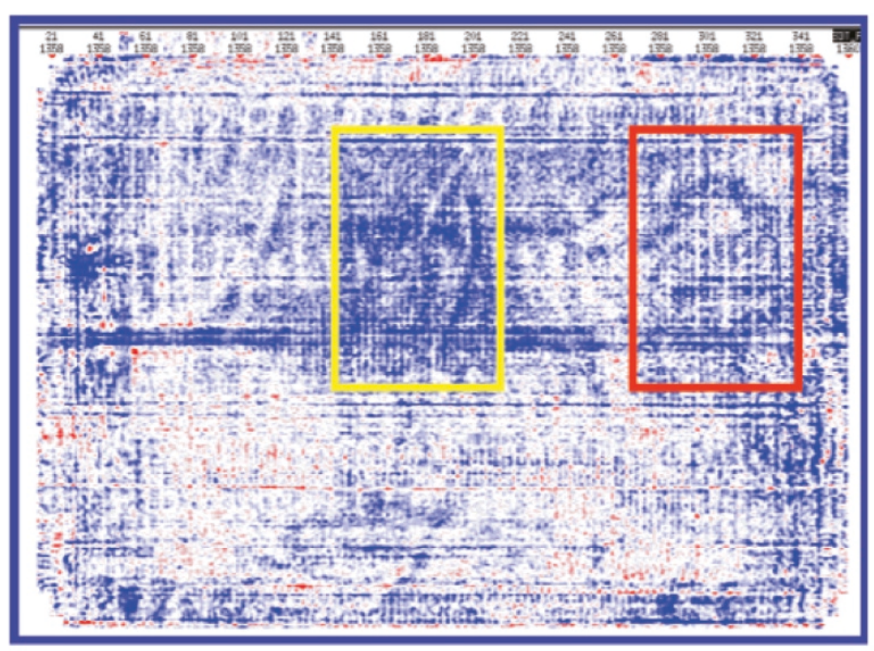

Narrow azimuth $1358 \mathrm{~ms}$

Fig. 4 Water-bottom reflection time slice of the seismic physical simulation experiment west-to-east rolling of observing shuttering. The belt width of the time slice above is $100 \mathrm{~m}$ (the distance between the two white belts), and that in the figure below is $60 \mathrm{~m}$, which are just equal to the half longitudinal rolling distance of shuttering.

Fig. 3 and Fig. 4 indicate that: (1) The stretching muting not only reduces the stacking fold of underground bins, but also creates non-uniform distribution of coverage, which leads to the shallow acquisition footprints that will possibly affect the imaging quality of shallow geological targets; (2) Different 3D geometries generate different acquisition footprints.

\section{Analysis of the target layer reflection acquisition footprints}

The burial depth of the target layer channel sand body is $2,000 \mathrm{~m}$, and the reflection time is $2.5 \mathrm{~s}$. We chose two pieces of time slice of each geometry to analyze and compare. Since the difference of travel times between the two sets of 3D data is $2 \mathrm{~ms}$ and wide azimuth is $2 \mathrm{~ms}$ later than narrow azimuth, the difference between the two kinds of time slice is $2 \mathrm{~ms}$. Fig. 5 shows the time slice of the target layer channel sand body imaging. Wide azimuth $2,530 \mathrm{~ms}$ and $2,542 \mathrm{~ms}$, and narrow azimuth $2,528 \mathrm{~ms}$ and $2,540 \mathrm{~ms}$ were chosen.

In the dereliction fan-shaped thin sand body seismic physical model experiment, we proved that: "Under the geological conditions of relatively smooth overburdens and little changed lateral velocities, 3D wide and narrow azimuth acquisition can basically obtain correct seismic imaging of underground targets using CMP stack migration processing and both imaging resolutions are equivalent" (Di et al, 2007). The channel sand body seismic physical model is simpler than the fan-shaped thin sand body model. The overburden of the channel sand body model is only three horizontal layers. We did the qualitative and quantitative analysis and compared the time slices between the wide/narrow azimuth geometries, and the result was similar to that of the fan-shaped thin sand body seismic physical model. This paper discusses the effects of the acquisition footprints under the precondition that the seismic imaging resolution of the wide and narrow azimuth observation is similar.

Acquisition footprints could only be seen in the background of the time slice. Some parts are clear while others are vague and all of the acquisition footprints are presented as longitudinal rolling belt traces. The belts of the wide azimuth geometry are wider, with the width of about $200 \mathrm{~m}$, but the belt of $100 \mathrm{~m}$ wide could also be seen in the enlarged drawing $(2,542 \mathrm{~ms})$. The belts of the narrow azimuth geometry are slender, with the width of $120 \mathrm{~m}$. Furthermore, the belt of $60 \mathrm{~m}$ wide could also be seen in the enlarged drawing $(2,542 \mathrm{~ms})$. Under the optimized wide/narrow azimuth geometries, although the time slices are presented in the footprints backgrounds, comparing the river bend part and the bifurcation part of the two kinds of slices carefully, we can discover that the acquisition footprints have little effect on the channel sand body imaging. 

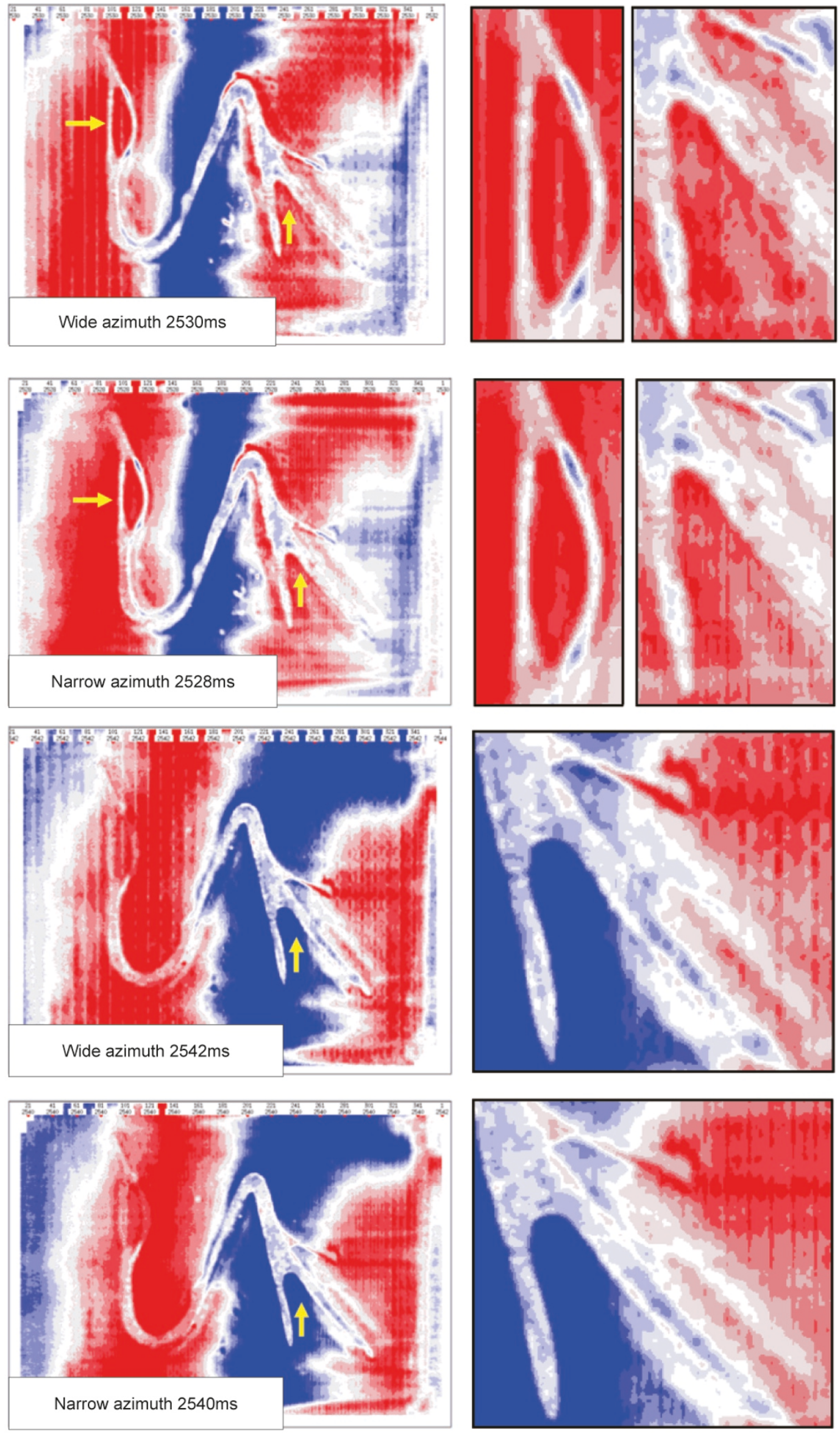

Fig. 5 Time slice of the channel sand body imaging in target layers

The right figures are the enlarged drawing of the part, which is indicated by the yellow arrow in the left figures 


\section{Conclusions}

Through the seismic physical simulation experiment, we have concluded that:

1) Discrete distribution of geometry and regular rolling of observation shuttering are the important generation mechanisms of acquisition footprints.

2) NMO stretching aberration processing will generate additional footprints, which are only located in the shallow or middle layers of the time slice, and possibly affect the imaging of shallow target layers, and have no influence on deep target imaging.

3) Different 3D geometries generate different acquisition footprints, so the acquisition footprint is an important issue in the optimization design of $3 \mathrm{D}$ geometries. Because the wide/narrow azimuth geometries in the experiment are both optimized, the footprints do not affect seismic imaging.

4) The seismic physical simulation technology has its advantages in the study of acquisition footprints.

\section{Acknowledgements}

The study is a project from the following: Heterogeneous formation geophysical response characteristics (973 Program, subject number: 2007CB209601) and Continental reservoir seismic physical model (CNPC Fundamental Research Projects, subject number: 06A10102).

\section{References}

Canning A and Gardner G H F. Reducing 3-D acquisition footprint for 3-D DMO and 3-D prestack migration. Geophysics. 1998. 63(4): $1177-1183$

Di B R, Sun Z X, Gu P C, et al. Analysis of influence of 3-D wide/ narrow geometry on seismic imaging (1): Acquisition study based on seismic physical simulation. Oil Geophysical Prospecting. 2007. 42(1): 1-6 (in Chinese)

Dong S T, Liu W L and Le J. Approach of suppressing acquisition footprint of 3-D seismic data. Oil Geophysical Prospecting. 2007. 42(1): 8-10 (in Chinese)

Feng K, He G H, Yin C, et al. Present situation and prospect of wideazimuth 3D inspection system. Journal of Southwest Petroleum University. 2006. 28(6): 24-28 (in Chinese)

Hou C F, Jiang L B and Gao S Q. 3-D geometry and acquisition footprints. Oil Geophysical Prospecting. 2007. 42(6): 611-615 (in Chinese)

Marfurt K J, Scheet R M, Sharp J A, et al. Suppression of the acquisition footprint for seismic sequence attribute mapping. Geophysics. 1998. 63(3): 1024-1035

Ma S H, Yang C C, Han X L, et al. The review of methods of acquisition footprint analysis and processing. Progress in Geophysics. 2008. 23(2): 500-507 (in Chinese)

Monk D. Pitfalls in seismic acquisition. The Leading Edge. 1999. 18(9): 1080-1083

Mou Y G and Pei Z L. Seismic numerical simulation of 3-D complex medium (Chapter VIII). Beijing: Petroleum Industry Press. 2005. 8-18 (in Chinese)

Qian G P, Kang J G and Ding C M. 3-D seismic exploration acquisition with wide-azimuth. Geophysical Prospecting for Petroleum. 2005. 44(2): 170-173 (in Chinese)

Schuster G T and Liu Z J. Seismic array theorem and rapid calculation of acquisition footprint noise. Geophysics. 2001. 66(6): 1843-1849

Tian M, Zhang M S, Wan C B, et al. Contrast research between wide azimuth seismic exploration and convention seismic exploration. Petroleum Geology \& Oilfield Development in Daqing. 2007. 26(6): 138-142 (in Chinese)

Xiong J L, Di B R, Yue Y, et al. Analysis of acquisition footprints based on seismic physical simulation. Oil Geophysical Prospecting. 2006. 41(5): 493-497 (in Chinese)

Yang H X. Advances in seismic data acquisition. Progress in Exploration Geophysics. 2003. 26(5-6): 463-468 (in Chinese)

Zhang J H, Zhang F, Zheng X G, et al. An overview of the methods and techniques for seismic acquisition footprints. Petroleum Instruments. 2007a. 21(5): 1-4 (in Chinese)

Zhang J H, Zhu H, Zheng X G, et al. Summary of wide-azimuth seismic exploration technique. Oil Geophysical Prospecting. 2007b. 42(5): 603-609 (in Chinese)

(Edited by Hao Jie) 